\title{
PUNTAS DE ARPÓN EN LA COSTA NORTE DE SANTA CRUZ: PRIMEROS ESTUDIOS DE PIEZAS RECUPERADAS EN ESTRATIGRAFÍA EN EL SITIO CUEVA DEL NEGRO
}

\author{
MARCELO BERETTA*, MIGUEL ZUBIMENDI**, MARIA CIAMPAGNA***, \\ PABLO AMBRÚSTOLO***** \& ALICIA CASTRO*
}

\begin{abstract}
RESUMEN
Este trabajo tiene como objetivo describir y analizar las características tecno-morfológicas de un conjunto de puntas de arpón halladas en estratigrafía en el sitio Cueva del Negro, ubicado en la costa norte de la provincia de Santa Cruz (Argentina). La mayoría de las piezas han sido manufacturadas en hueso, aunque un ejemplar fue confeccionado sobre madera. Se utilizará, con fines comparativos, información bibliográfica sobre otros ejemplares recuperados en la Patagonia continental. Se discutirá la presencia de las puntas de arpón en el registro arqueológico como indicadores de tecnologías con diseños especializados para la explotación de recursos marinos en la costa norte de Santa Cruz.
\end{abstract}

PALABRAS CLAVE: arpón, cazadores recolectores, recursos marinos, costa norte de Santa Cruz.

HARPOON HEADS ON THE NORTH COAST OF SANTA CRUZ: INITIAL STUDIES AND ANALYSIS OF PIECES RECOVERED IN STRATIGRAPHY AT SITE CUEVA DEL NEGRO

\section{ABSTRACT}

The aim of this paper is to describe and to analyse the techno-morphological features of a sample of harpoon heads founded in stratigraphic context at Cueva del Negro site at the northern coast of Santa Cruz Province (Argentina). Those pieces have been elaborated on bone but a fragment on wood has been founded associate to them. Comparative analysis will be performed with bibliographic information about

\footnotetext{
* Museo de La Plata, Facultad de Ciencias Naturales y Museo, Universidad Nacional de La Plata. Email: jmb_zooarqueologia@ yahoo.com.ar / aliciacastro52@gmail.com.

** CONICET; Unidad Académica Caleta Olivia, Universidad Nacional de la Patagonia Austral, y Museo de La Plata, Facultad de Ciencias Naturales y Museo, Universidad Nacional de La Plata. Paseo del Bosque s/n; Email: mikelzubimendi@gmail.com.

*** Becaria doctoral CONICET; Museo de La Plata, Facultad de Ciencias Naturales y Museo, Universidad Nacional de La Plata. Email: mlciampagna@gmail.com.

***** Becario Postdoctoral CONICET; Museo de La Plata, Facultad de Ciencias Naturales y Museo, Universidad Nacional de La Plata. Email: pambrustolo@hotmail.com.
} 
other samples found at continental Patagonia and its presence within the archaeological record from northern coast of Santa Cruz, will be discussed considering those instruments as a product of specialized technology oriented to the hunting of littoral resources.

KEY WORDS: harpoon, hunters and gatherers, marine resources, north coast of Santa Cruz.

\section{INTRODUCCIÓN}

En este trabajo presentamos siete puntas o cabezales de arpón (sensu Caggiano 1977), recuperados en estratigrafía en el sitio conchero Cueva del Negro (costa norte de Santa Cruz). Se realizará una descripción morfológica de los hallazgos y un análisis tanto de la materia prima como de las marcas antrópicas que presentan, con la finalidad de interpretar las técnicas de manufactura y la funcionalidad de las piezas. También se discutirá la presencia de los arpones en contextos arqueológicos de la costa Norte de Santa Cruz como indicadores de conductas asociadas a prácticas de caza y consumo de pinnípedos.

Las piezas mencionadas corresponden a los primeros hallazgos de este tipo de instrumento en contextos estratigráficos de la Patagonia continental argentina, ya que los antecedentes existentes se relacionan con ejemplares recolectados asistemáticamente en superficie por coleccionistas o aficionados, por lo que sólo se consignaron datos muy generales y escasos (Outes 1916; Vignati 1930; Molina 1967-1970, entre otros).

En relación con los arpones de hueso en la Patagonia continental, E. Moreno (2009) en su tesis doctoral realizó una recopilación bibliográfica y el relevamiento de colecciones privadas de la costa norte de Santa Cruz. Analizó su variabilidad morfológica y desarrolló un estudio comparativo de su distribución geográfica en relación con los rompecráneos, artefactos posiblemente utilizados para la caza de pinnípedos (Moreno et al. 2000). Moreno discute el uso probable de los arpones para la captura de los mencionados recursos costeros. Estos datos serán utilizados para contextualizar la discusión de los nuevos hallazgos de Cueva del Negro a fin de obtener un marco amplio de discusión sobre este tipo de tecnología para la zona de estudio.

Es importante señalar que la mayoría de los restos óseos arqueofaunísticos recuperados en el sitio Cueva del Negro corresponden a pinnípedos de diferentes rangos etarios (Beretta et al. 2011; Zubimendi et al. 2012). En este trabajo consideraremos, al igual que Moreno (2009), que el uso de los arpones se relaciona con la captura de grandes mamíferos marinos, aunque no necesariamente por medio del uso de embarcaciones, como es el caso de las canoas utilizadas por las poblaciones del canal Beagle o los canales septentrionales chilenos.

\section{Antecedentes de puntas de arpón en la Patagonia insular y continental}

Los arpones en el registro etnohistórico

En las fuentes etnohistóricas de los archipiélagos de Patagonia sur y occidental se menciona el uso de arpones para la caza de pinnípedos entre los grupos chonos, alacalufes y yaganes. Barros Valenzuela (1931) por ejemplo, destaca el uso de arpones de hueso entre los chonos de Chiloé y la península de Taitao, registrando una punta multidentada de hueso de cetáceo de $300 \mathrm{~mm}$ de largo. Cárdenas et al. (1991) menciona, para la caza de pinnípedos entre los alacaluf, el uso de arpones bidentados de hueso de cetáceo con dientes opuestos.

A partir de estudios etnográficos en Tierra del Fuego, Orquera \& Piana (1999a) distinguen dos tipos básicos de arpones: los de punta fija y los de punta móvil o separable. Asimismo, en base al análisis de caracteres morfo-funcionales, plantean que hay dos formas de definir un arpón: por la cantidad de dientes, es decir, por la capacidad de retención que posee el arma una vez clavada en la presa; y por la movilidad o no de la punta respecto del mango, es decir, por la posibilidad de que la punta se separe en el momento de herir a la presa y de esta forma disminuyan los riesgos de fractura de la misma (Orquera \& Piana 1999b).

Los arpones de punta fija se destinaban principalmente para la pesca y la caza de aves, siendo utilizados para herir y matar directamente al animal 
(Legoupil 2003). Se caracterizan por poseer una mayor cantidad de dientes (puntas multidentadas), conformando una hilera de seis a veinte dientes pequeños. Estas puntas de arpón poseen una mayor longitud y una espiga más delgada, con dimensiones que van desde 41 a $130 \mathrm{~mm}$ de largo máximo (Orquera \& Piana 1999b).

Las puntas móviles de arpón se caracterizan por poseer un diente (monodentado), siendo mucho menos frecuente los que poseen dos. La base o espaldón se presenta ensanchada, y es denominada, por Orquera y Piana espaldón simple.Los estudios indican que este tipo de punta o cabezal era ajustado flojamente al astil; de esta forma, una vez que el cabezal se clavaba en la presa, el mango se desprendía y quedaba unido por una correa de cuero, generalmente de pinnípedo. De esta forma, la resistencia en el agua producida por el astil dificultaba la huida de la presa, produciéndole una significativa pérdida de sangre, y el astil, a manera de flotante, señalaba la posición del animal (Orquera et al. 1977:82; Orquera \& Piana 1999b; Legoupil 2003). En este sentido, estas armas estaban confeccionadas para la caza de pinnípedos desde canoas o, en algunos casos, para la captura de ballenas.

Los arpones en el registro arqueológico

Las puntas de arpón fijas multidentadas han sido recuperadas en sitios arqueológicos desde el seno de Reloncaví en la comuna de Puerto Montt hasta en los sitios del canal Beagle, en Tierra del Fuego (Ortíz Troncoso 1975; Legoupil 1997, 2003; Gaete et al. 2004; San Román 2005; Orquera \& Piana 1999b).

Las puntas de arpón móviles monodentadas también se han recuperado en sitios de la región del estrecho de Magallanes, el canal Beagle y la Patagonia continental argentina (entre otros, Ortiz Troncoso 1971; Orquera \& Piana 1999b; Legoupil 2003; Moreno 2009; Buc \& Cruz 2012). Se han registrado medidas de entre 160 y $200 \mathrm{~mm}$ de largo en el canal Beagle (Orquera \& Piana 1995), mientras que Scheinsohn (1998) registró arpones con medidas entre 145 y 272 mm de largo; en el sitio Ponsonby, Leogupil (2003) recuperó una punta de arpón de espaldón simple monodentado de $400 \mathrm{~mm}$. Los materiales óseos que generalmente se utilizan para la confección de estos instrumentos son de animales marinos; principalmente mandíbulas de ballenas y costillas de pinnípedos. Los huesos de guanaco usados para tal efecto, son menos comunes (Orquera \& Piana 1999a; Scheinsohn 1998).

Por otra parte, se registraron en sitios arqueológicos de los archipiélagos chilenos y canal Beagle, puntas de arpón monodentadas con dos protuberancias basales (cruciformes) decoradas, manufacturadas sobre hueso de animales marinos. (Ortiz Troncoso 1975; Orquera et al. 1977; Legoupil 1997; San Román 2005 y Torres \& Ruz 2011).

En la Patagonia continental argentina, se han registrado hasta el momento 26 puntas de arpón con espaldón simple monodentadas, en varias localidades arqueológicas de la costa central (Moreno 2009). Las primeras menciones provienen de Cabo Blanco (por ejemplo, Outes 1916 y Vignati 1930), en el extremo sur del golfo San Jorge, mientras que son más abundantes en la costa de la bahía de los Nodales y Punta Medanosa (Molina 1967-1970). Recientemente se han hallado también dos arpones de este tipo en la localidad arqueológica Punta Entrada (Buc \& Cruz 2012). Todas estas piezas -salvo las de Punta Entrada- han sido recuperadas por coleccionistas, por lo que carecen de mayores datos de procedencia y contexto de hallazgo.En los casos en que se pudo determinar la materia prima, se estableció que fueron manufacturados a partir de huesos de pinnípedos o cetáceos (Fig. 1).

Nuevos hallazgos de arpones en la costa norte de Santa Cruz

El sitio Cueva del Negro, ubicado en la costa norte de la provincia de Santa Cruz, es un abrigo rocoso emplazado en el frente de un afloramiento de rocas porfíricas. Consta de un alero y un recinto subcircular profundo y oscuro. Este último presenta evidencias de alteraciones por saqueo (Beretta et al. 2011), mientras que en el sector del alero la secuencia estratigráfica -conformada por un conchero de gran potencia-se halla intacta (Zubimendi et al. 2012).

Los cabezales de arpón provienen de las cuadrículas 1 y 3 del alero y del recinto oscuro. En la cuadrícula 1 se recuperaron cuatro puntas asociadas a tres fechados radiocarbónicos (Zubimendi et al. 2012): uno sobre un húmero de pinnípedo (antigüedad corregida de $1220 \pm 80$ años AP), otro sobre una hemimandíbula de pinnípedo (antigüedad 


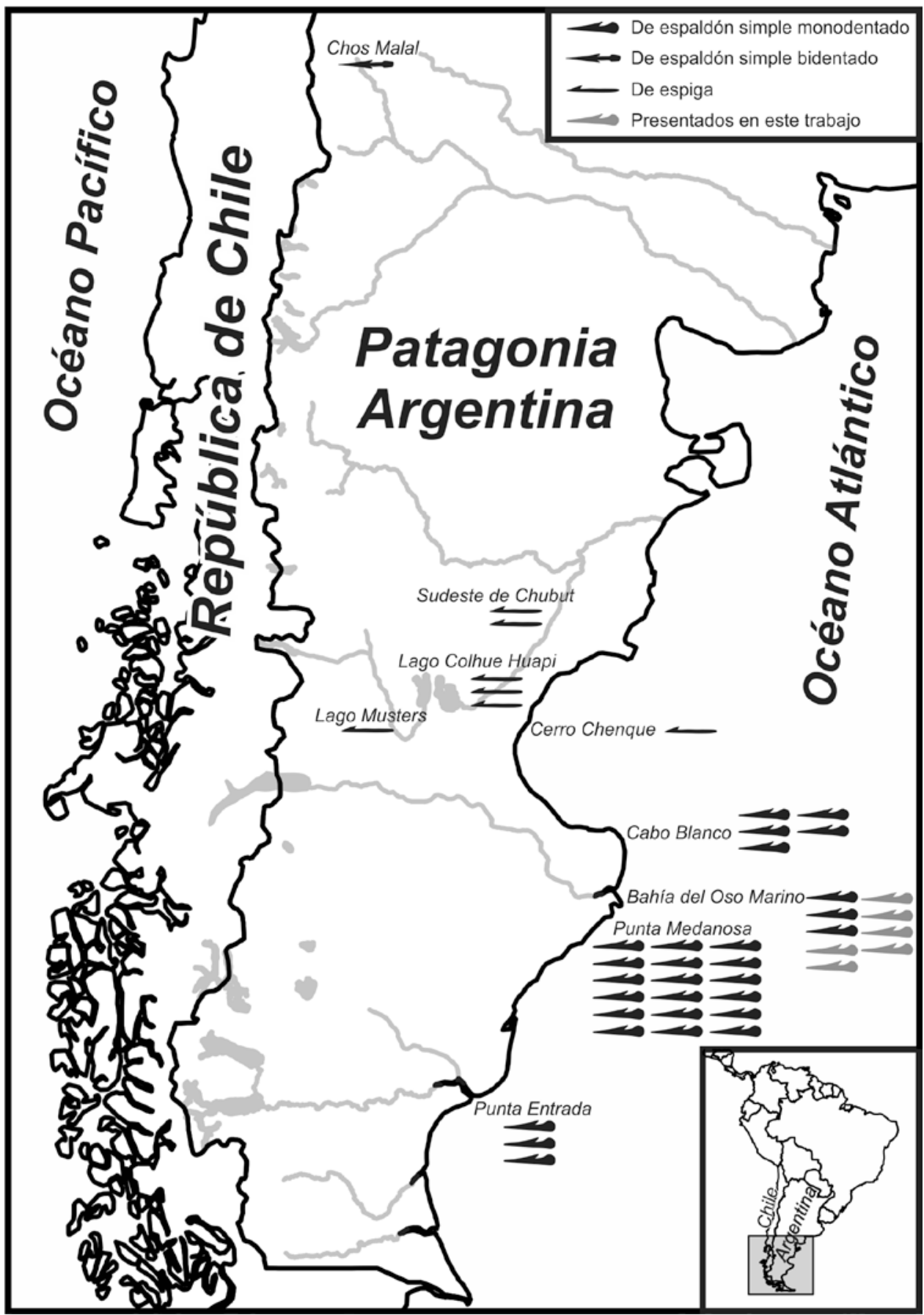

Fig. 1. Distribución espacial de los arpones de hueso existentes en la Patagonia continental argentina. En gris los arpones presentados en este trabajo. 
corregida de $1290 \pm 50$ años AP) y un tercer fechado sobre carbones provenientes de una estructura de combustión (1340 \pm 60 años AP). En la cuadrícula 3 se recuperaron dos puntas de arpón, las cuales no cuentan con fechados asociados; sin embargo por las características contextuales de depositación se presupone contemporaneidad. En el recinto oscuro, en contextos alterados por saqueo, se recuperó otra punta de arpón confeccionada sobre madera perteneciente al género Berberis (Capparelli et al. 2009; Zubimendi et al. 2012).
Descripción y análisis de las puntas de arpón

Todas las piezas recuperadas son, según las características morfológicas, puntas de arpón de espaldón simple monodentadas, correspondientes a las utilizadas en arpones de punta móvil o separable (sensu Orquera \& Piana 1999a). En cuanto a las dimensiones, están consignadas en forma comparativa en la Tabla 1. Con el propósito de establecer similitudes y diferencias entre los distintos ejemplares, tanto en hueso como en madera, se realizó un análisis morfológico (Tabla 2). Con excepción de un ejemplar

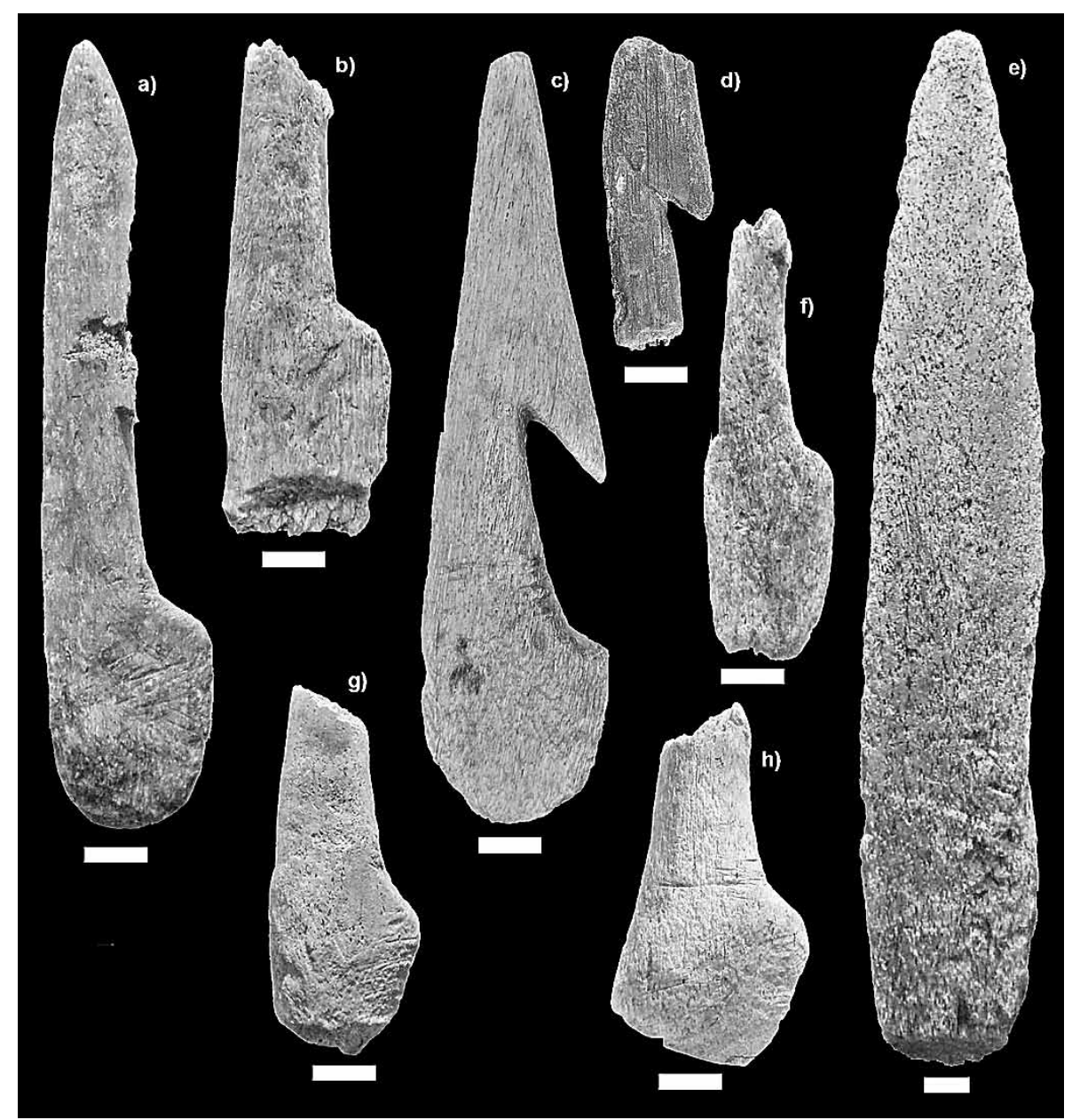

Fig. 2. Vista de los cabezales de arpón recuperados en el sitio Cueva del Negro. Referencias: a) arpón que presenta espolón y punta, pero con ausencia del diente producto de una fractura (pieza 2); b) espaldón, fragmento central y posterior de arpón (pieza 6); c) arpón monodentado entero (pieza 1); d) fragmento central de arpón de madera de calafate (Berberis sp.; pieza 7); e) probable preforma de arpón (Pieza 8); f) espaldón, fragmento central y posterior de arpón (pieza 4); g) espaldón, fragmento posterior de arpón (pieza 5); y h) espaldón, fragmento posterior de arpón (pieza 3). En todos los casos la barra blanca equivale a $1 \mathrm{~cm}$. 
Tabla 1. Cuadro comparativo de las diferentes dimensiones de cada una de las piezas de hueso (1 - 6) y madera (7), correspondiente a puntas de arpón enteras y fragmentadas halladas en estratigrafía. Los valores que se observan en la tabla fueron tomadas en milímetros. Las medidas de cada una de las porciones fueron realizadas desde la parte posterior o proximal hacia la anterior o distal. Las dos cuantías que se observan en los distintos segmentos de la punta de arpón, tanto en altura como en espesor, corresponden a los valores mínimo y máximo registrados sobre cada parte de la pieza. Referencias: esp.= espaldón; fg.= fragmento.

\begin{tabular}{|c|c|c|c|c|c|c|c|c|}
\hline \multicolumn{2}{|c|}{ Número de Pieza } & 1 & 2 & 3 & 4 & 5 & 6 & 7 \\
\hline \multicolumn{2}{|c|}{ Estado de la Pieza } & $\begin{array}{c}\text { Entera } \\
\text { (Fig. 2.c) }\end{array}$ & $\begin{array}{c}\text { incompleta } \\
\text { (Fig. 2.a) }\end{array}$ & $\begin{array}{l}\text { esp.+fg. fuste } \\
\text { (Fig. 2.h) }\end{array}$ & $\begin{array}{c}\text { esp.+ } \\
\text { fuste } \\
\text { (Fig. 2.f) }\end{array}$ & $\begin{array}{l}\text { esp.+fg. } \\
\text { fuste } \\
\text { (Fig. 2.g) }\end{array}$ & $\begin{array}{c}\text { esp.+ fuste } \\
\text { (Fig. 2.b) }\end{array}$ & $\begin{array}{l}\text { fg. fuste + } \\
\text { fg. aguja } \\
\text { (Fig. 2.d) }\end{array}$ \\
\hline \multirow{4}{*}{ Longitud } & Total & 110,87 & 122,9 & - & - & - & - & - \\
\hline & Espaldón & 27,24 & 35,66 & 27,3 & 37,77 & 28,05 & 35,3 & - \\
\hline & Fuste & 32,15 & 26,84 & - & 25,47 & - & 28,13 & 22,3 \\
\hline & Diente & 51,48 & 60,4 & - & - & - & - & 24,55 \\
\hline \multirow{3}{*}{ Ancho } & Espaldón & $14,77-26,86$ & $17,93-25,25$ & $23,38-27,30$ & $\begin{array}{l}14,56- \\
20,21\end{array}$ & $\begin{array}{l}12,76- \\
23,10\end{array}$ & $24,27-25,74$ & - \\
\hline & Fuste & $21,42-12,74$ & $17,82-14,11$ & $18,23-?$ & $\begin{array}{c}12,04- \\
9,58\end{array}$ & $15,91-?$ & $16,73-13,82$ & $?-9,68$ \\
\hline & Diente & $24,50-6,52$ & $?-1,43$ & - & - & - & - & $16,04-?$ \\
\hline \multirow{3}{*}{ Espesor } & Espaldón & $6,40-16,50$ & $5,48-11,85$ & $16,22-17,53$ & $\begin{array}{c}6,64- \\
9,55\end{array}$ & $8,38-11,45$ & $7,28-11,43$ & - \\
\hline & Fuste & $16,50-14,69$ & $11,85-11,50$ & $17,53-?$ & $\begin{array}{l}9,55- \\
9,14 \\
\end{array}$ & $11,45-?$ & $11,43-11,71$ & $?-6,70$ \\
\hline & Diente & $14,69-4$ & $11,50-1,66$ & - & - & - & - & $6,70-?$ \\
\hline \multicolumn{2}{|c|}{ Sobresaliencia del diente } & 17,18 & - & - & - & - & - & 7,39 \\
\hline
\end{tabular}

(Fig. 2c, pieza 1), la mayoría de las puntas de arpón se encuentran fracturadas: cuatro están representadas por su porción proximal o espaldón (Fig. 2h, f, g y b piezas 3, 4, 5 y 6), una por la parte medial o fuste (Fig. 2d, pieza 7) y otra presenta una fractura en su diente (Fig. 2a, pieza 2).

En la Tabla 2 se observa que la materia prima de la mayoría de las puntas de arpón corresponde a huesos (mandíbula y costilla) de cetáceos, salvo la pieza 4 (Fig. 2.f) confeccionada sobre costilla o metapodio de pinnípedo. La disposición y la compactación de las fibras del tejido esponjoso del hueso (observadas a lupa binocular), como así también el peso de la pieza, fueron las variables que se utilizaron para determinar el taxón correspondiente. Por otro lado, la forma estructural del tejido esponjoso, la densidad, las dimensiones y la presencia-ausencia de curvatura en toda la longitud, fueron los rasgos que determinaron la unidad anatómica. En el caso de la pieza 1 (Fig. 2.c) las dimensiones permiten suponer que se trata de una mandíbula de ballena. En cambio, la presencia de cierto grado de curvatura en toda su longitud en la pieza 2 (Fig. 2.a), permite afirmar que fue elaborada sobre una costilla. Para determinar la unidad anatómica de los fragmentos de puntas de arpón se utilizaron los primeros dos criterios. De acuerdo a los lineamientos mencionados por Miotti (1990-1992), la pieza 2 presenta una fractura en estado fresco, ya que la misma es longitudinal, de ángulo agudo y la superficie de fractura es recta y de color similar al resto de la pieza. Por otra parte, se observan marcas de corte antrópicas tipo V (Binford 1981; Lyman 1994), oblicuas y perpendiculares al eje de la pieza, en el espaldón y el fuste de cuatro cabezales de arpón. La preservación de la mayoría de las piezas es buena y muy buena. Solo la pieza 4 presenta una meteorización de grado 3 (sensu Mengoni Goñalons 1999; Borella 2004).

La pieza 7 corresponde al fragmento de arpón confeccionado sobre Berberis sp. (Capparelli et al. 2009). La punta del arpón es de tamaño pequeño y posee un solo diente prominente (Fig. 2.d). Ambos extremos del instrumento se hallan fragmentados, sólo están presentes gran parte del fuste y la primera porción de la aguja. Cabe señalar que presenta rastros de alteración térmica en la aguja y en la porción proximal. Sobre la cara lateral izquierda del fuste se observan marcas de corte tipo 
Tabla 2. Cuadro comparativo de las diferentes características morfológicas observadas en cada una de las piezas confeccionadas en hueso.

\begin{tabular}{|c|c|c|c|c|c|c|}
\hline Número de Pieza & 1 & 2 & 3 & 4 & 5 & 6 \\
\hline $\begin{array}{c}\text { Según cantidad de } \\
\text { dientes }\end{array}$ & Monodentado & Monodentado & - & - & - & - \\
\hline $\begin{array}{c}\text { Forma del Corte } \\
\text { transversal de la } \\
\text { aguja }\end{array}$ & $\begin{array}{l}\text { Oval de bordes } \\
\text { redondeados }\end{array}$ & $\begin{array}{c}\text { Oval de bordes } \\
\text { agudos }\end{array}$ & - & - & - & - \\
\hline Forma del espaldón & Redondeada & Romo & Cuadrangular & Espatuliforme & Redondeado & Cuadrangalar \\
\hline $\begin{array}{l}\text { Técnica de } \\
\text { confección }\end{array}$ & $\begin{array}{l}\text { Raspado y } \\
\text { pulido }\end{array}$ & $\begin{array}{l}\text { Raspado y } \\
\text { pulido }\end{array}$ & $\begin{array}{l}\text { Raspado y } \\
\text { pulido }\end{array}$ & - & $\begin{array}{l}\text { Raspado y } \\
\text { pulido }\end{array}$ & $\begin{array}{l}\text { Raspado y } \\
\text { pulido }\end{array}$ \\
\hline Materia prima & $\begin{array}{l}\text { Mandíbula de } \\
\text { cetáceo }\end{array}$ & $\begin{array}{l}\text { Costilla de } \\
\text { cetáceo }\end{array}$ & $\begin{array}{l}\text { Mandíbula de } \\
\text { cetáceo }\end{array}$ & $\begin{array}{l}\text { Hueso de } \\
\text { pinnípedo }\end{array}$ & $\begin{array}{l}\text { Mandíbula de } \\
\text { cetáceo }\end{array}$ & $\begin{array}{l}\text { Costilla de } \\
\text { cetáceo }\end{array}$ \\
\hline Marcas antrópicas & Corte tipo $\mathrm{V}$ & Corte tipo $\mathrm{V}$ & Corte tipo V & $\begin{array}{c}\text { No se } \\
\text { observan }\end{array}$ & Corte tipo V & Ausentes \\
\hline $\begin{array}{c}\text { Disposición de las } \\
\text { marcas }\end{array}$ & Perpendiculares & Oblicuas & Oblicuas & - & Oblicuas & - \\
\hline $\begin{array}{c}\text { Ubicación de las } \\
\text { marcas }\end{array}$ & $\begin{array}{l}\text { Espaldón y } \\
\text { fuste }\end{array}$ & Espaldón y fuste & Espaldón & - & Espaldón & - \\
\hline Meteorización & Grado 0 & Grado 0 & Grado 0 & Grado 3 & Grado 0 & Grado 0 \\
\hline
\end{tabular}

V oblicuas a las fibras de la madera. También posee estrías poco profundas, paralelas a las fibras, que se observan a lo largo del fragmento de arpón.

En asociación espacial a las puntas de arpón halladas en la capa 3 de la cuadricula 3, se recuperó un artefacto de hueso que por sus características morfológicas y modificaciones antrópicas probablemente corresponda a una preforma de punta de arpón (Pieza 8; Fig. 2.e). Los caracteres diagnósticos mencionados previamente sugieren que este artefacto está confeccionado sobre una costilla de pinnípedo. La misma presenta un extremo romo y otro con terminación punzante. Posee una longitud de $220 \mathrm{~mm}$ desde la fractura (extremo proximal) a la punta (extremo distal). La altura varía entre 27,21 mm en la porción proximal, $35,78 \mathrm{~mm}$ en la porción medial y 7,86 $\mathrm{mm}$ en la porción distal. Su espesor es de 15,05 mm, 15,73 $\mathrm{mm}$ y $5,74 \mathrm{~mm}$ en las porciones proximal, medial y distal respectivamente. El extremo romo se produjo por una fractura contundente de origen antrópico $y$, por sus características, se efectuó sobre hueso en estado fresco. Se observan marcas de corte de tipo $\mathrm{V}$ sobre una de las caras laterales externa de la costilla y de los tipos $\mathrm{V}$ y $\mathrm{Z}$ sobre los bordes superior e inferior de la pieza. Por otro lado, la cara lateral interna muestra la ausencia de tejido compacto, sin poder precisar si se debe a una alteración antrópica o de origen natural. Esta pieza presenta una meteorización de grado 3 .

\section{DISCUSIÓN}

El hallazgo de las puntas de arpón en el sitio Cueva del Negro cobra relevancia debido a que su asociación estratigráfica primaria permite una interpretación contextual de las mismas.

El registro de piezas con fracturas en estado seco podría deberse a que las puntas se fracturaron durante su utilización y fueron posteriormente desechadas (descarte predeposicional), o bien tratarse de piezas descartadas y fracturadas por procesos posdeposicionales. Si se tratara de este último caso, sería esperable hallar asociados tanto espaldones como agujas, sin embargo los fragmentos de puntas de arpón recuperados en el registro arqueológico de Cueva del Negro corresponden únicamente a espaldones. Esto podría estar relacionado con la mecánica de fractura de las puntas de arpón producida durante la caza de animales marinos de gran porte, como los pinnípedos. En este sentido, cuando la punta de hueso se inserta en la carne del animal, éste trata de huir y ejerce una fuerza contrapuesta con la del cazador, ocasionando posiblemente el quiebre de 
dicha punta a la altura del fuste o del diente de la aguja. El fragmento distal de la punta quedaría en el cuerpo del animal y el proximal permanecería atado con la cuerda al astil. El descarte del espaldón en el recambio de una nueva punta, podría explicar la presencia de dichos fragmentos en el sitio. La ausencia de extremos distales en el sitio podría deberse a varias circunstancias, una posibilidad es que dichos fragmentos quedaran abandonados fuera del sitio; o bien en casos de predación no exitosa, que el animal haya escapado con el fragmento de arpón clavado. Por otra parte, cabe mencionar la posibilidad de que la ausencia de las mismas se relacione con un problema de muestreo.

Se han registrado piezas con fracturas en estado fresco, como la pieza 2, las que sugieren que se habrían producido durante el proceso de formatización de los instrumentos, constituyendo la causa de descarte. Esto permitiría plantear la posibilidad de la manufactura local de las puntas de arpón, teniendo en cuenta la combinación de diversas técnicas como el rebaje y pulido, evidenciado por la presencia de cortes y hendiduras a nivel micro y macroscópico. Sin embargo, algunos autores sugieren que el intercambio explicaría la presencia de puntas de arpones de hueso en este sector de la costa patagónica (Gómez Otero et al. 1998). En este caso, consideramos como más probable la manufactura local debido a que las morfologías de las puntas de arpón recuperadas en la Cueva del Negro difieren de las registradas en los canales septentrionales chilenos y el canal Beagle. Refuerza esta posibilidad el hecho de que las materias primas necesarias para la confección de las piezas están disponibles en abundancia debido a la gran cantidad de loberías existentes en el área (Carrara 1952; Schiavini et al. 1999) y el varamiento ocasional de cetáceos.

La pieza 8 es interpretada como una posible preforma, correspondiente a una fase inicial de la confección de un cabezal de arpón. En la misma se observaron alteraciones antrópicas y posee volumen y densidad suficientes como para soportar el desgaste de la formatización de una punta de arpón completa. El hallazgo de esta pieza reforzaría la hipótesis de la manufactura local de los cabezales de arpón.

En términos generales, las dimensiones de los ejemplares recuperados hasta el momento son menores que las registradas por Legoupil (2003) para los canales chilenos, por Orquera \& Piana (1999a) para el canal Beagle, y por Scheinsohn (1998) para la costa de la Isla Grande de Tierra del Fuego. Esta diferencia dimensional, podría estar relacionada con las características de la materia prima tanto de la punta de arpón como del astil. Es decir, si la punta es de menor tamaño, el astil también debería serlo. Considerando la probable manufactura local de estos instrumentos, la ausencia de material vegetal leñoso con tramos rectos mayores a $60 \mathrm{~cm}$ en el área de costa norte de Santa Cruz (Cabrera 1971), podría haber determinado el tamaño de los cabezales de arpón.

En relación con la materia prima de los cabezales óseos, la mayoría de los ejemplares se confeccionaron sobre huesos de cetáceo, más precisamente con las costillas y mandíbulas de dichos animales. En cambio, una de las piezas, al igual que la preforma, fueron elaboradas sobre costilla de pinnípedo. Según Scheinsohn (2005), estas partes del esqueleto proporcionan una buena superficie para la manufactura de las puntas y ofrecen cierto grado de elasticidad que evita las quebraduras de las puntas móviles de los arpones.

El fragmento medial de punta de arpón de madera (Pieza 7) presenta dimensiones menores a las puntas óseas. En este sentido, Molina (1967-1970:178) menciona un dato aportado por E. Chapoala, un informante indígena de la provincia de Santa Cruz, el cual hace referencia a que antiguamente se empleaban arpones de madera para la pesca en pozones. Esto sugiere que el arpón de madera podría haber sido utilizado para la captura de otros tipos de recursos como los peces (Emperaire 2002).

Los arpones de espaldón simple, en el caso de la costa continental de Patagonia argentina, han sido recuperados exclusivamente en el sector central de la misma, más específicamente entre Cabo Blanco y la desembocadura del río Santa Cruz (Gómez Otero et al. 1998; Moreno 2009). Se ha planteado que la distribución de arpones estaría vinculada a sectores de costa donde se registran sitios arqueológicos con abundantes restos óseos de pinnípedos (Moreno 2009) y próximos a loberías (Castro et al. 2003; Zubimendi et al. 2004). Asimismo, a excepción de los arpones recuperados en Punta Quilla y Punta Entrada, estos instrumentos se asocian a sectores de costa irregulares con afloramientos rocosos ignimbríticos e islas en las que existieron (Carrara 1952), o existen hoy en día, loberías (Schiavini et 


\section{al. 1999).}

Otros instrumentos con una distribución estrictamente litoral, asociados también a las loberías, son los rompecráneos (Moreno et al. 2000; Moreno 2009). A partir del análisis de colecciones se registró la asociación de arpones y rompecráneos en las mismas localidades arqueológicas de la Patagonia central. En cuanto a la asignación cronológica de los rompecráneos, cabe mencionar que éstos han sido recuperados en estratigrafía en ocupaciones datadas en el Holoceno tardío final (Castro et al. 2007), asociados a gran cantidad de restos faunísticos de pinnípedos.

La asociación entre arpones, rompecráneos y pinnípedos, posiblemente se dé en el marco de un mismo sistema tecnológico de captura. Estos dos tipos de instrumentos son los únicos que se diferencian del repertorio tecnológico que constituyen los conjuntos instrumentales conocidos para el Holoceno tardío en la región patagónica argentina (ver Orquera 1987). Ambos están manufacturados a partir de materias primas locales. Más allá de las diferencias en las técnicas y la cantidad de energía implicada en la manufactura de cada uno -probablemente mayor en el caso de los arpones-, presentan diseños muy específicos e implican un profundo conocimiento de las características de las especies explotadas para poder hacer un uso efectivo de los mismos.

\section{CONSIDERACIONES FINALES}

En el sitio Cueva del Negro se hallaron por primera vez en estratigrafía, en la costa atlántica de Patagonia, arpones de espaldón simple en muy buen estado de conservación, lo que permitió avanzar en el estudio de este tipo de instrumentos muy escasos en el registro arqueológico de la Patagonia continental.

Los ejemplares fueron manufacturados en su mayoría a partir de costillas y mandíbulas de cetáceo, una pieza sobre una costilla de pinnípedo y otra a partir de leño de Berberis sp. La disponibilidad de materia prima ósea y vegetal, así como la presencia de una preforma y de una pieza fracturada en estado fresco podrían indicar la manufactura local de los mismos.

En relación con la distribución de los arpones descriptos en la revisión de E. Moreno
(2009), observamos que este tipo de instrumentos está asociado a sectores de costa, donde se registran sitios arqueológicos con abundantes restos óseos de pinnípedos, localizados próximos a grandes loberías existentes en el pasado y, en su mayoría, cercanos a costas rocosas con islas e islotes adyacentes. Tendencias similares son registradas respecto de los instrumentos líticos denominados rompecráneos. La importancia de ambos instrumentos y su asociación con contextos vinculados con una intensiva explotación de pinnípedos, sugieren una estrategia de caza desde la costa, aunque sin el uso de canoas, a diferencia de los antecedentes existentes en la costa chilena y de Tierra del Fuego. Si bien se sugiere que estos artefactos estarían asociados a la caza de pinnípedos, es importante mencionar que el fragmento de arpón de madera podría haber sido utilizado para la pesca.

Los fechados de la secuencia estratigráfica de Cueva del Negro indican que los cabezales de arpón de la costa norte de Santa Cruz pertenecen a momentos del Holoceno tardío, más específicamente hace ca. 2000 y 1000 años AP. En estos momentos, este sector de costa habría sido utilizado con mayor intensidad (Ambrústolo 2011; Castro et al. 2003; Moreno 2009; Zubimendi et al. 2004, 2005 y 2012), con una ocupación efectiva del espacio (Borrero 19941995) por parte de grupos cazadores recolectores en el marco de una economía de amplio espectro en la zona de la bahía del Oso Marino (Beretta et al. 2011) y con tecnologías especializadas para la explotación de los recursos marinos.

\section{AGRADECIMIENTOS}

Quisiéramos agradecer a todo el equipo de investigación del proyecto Arqueología de la Costa Norte de Santa Cruz que colaboraron en distintas instancias de este trabajo: L. Mazzitelli, H. Hammond, L. Zilio, V. Trola, L. Corinaldessi, S. Bogan y A. Herrera; así como a E. Moreno, quien leyó una versión preliminar de este trabajo. También queremos agradecer las siguientes empresas, instituciones y personas gracias a quienes pudimos realizar los trabajos de campo: YPF S. A., PROSEPET, Vialidad y Consejo Agraria de la Provincia de Santa Cruz, Municipalidad y Museo Municipal Mario Brozoski de Puerto Deseado, así como a G. y C. Jenkins. 


\section{BIBLIOGRAFÍA}

Ambrústolo, P. (2011). Estudio de las estrategias de aprovisionamiento y utilización de los recursos líticos por grupos cazadores-recolectores en la costa norte de Santa Cruz (Patagonia Argentina). Doctorado, Universidad Nacional de La Plata.

Barros Valenzuela, A. (1931). Chonos. En Aborígenes australes de América. Santiago: Lord Cochrane.

Beretta, M., L. Corinaldessi \& A. Castro. (2011). Recursos marinos versus recursos terrestres: análisis arqueofaunístico en el sitio Cueva Del Negro, costa norte de Santa Cruz. Revista Arqueología 17, 1-23.

Binford, L. (1981). Bones: Ancient Men and Modern Myths. New York: Academic Press.

Borella, F. (2004). Tafonomía regional y estudios arqueológicos de cetáceos en Tierra del Fuego y Patagonia meridional. Oxford: Archaeopress (BAR S1257).

Borrero, L. A. (1994-1995). Arqueología de la Patagonia. Palimpsesto. Revista de Arqueología 4, 9-69.

Buc, N. \& Cruz I. (2012). El aprovechamiento de la fauna como instrumental óseo en la costa al sur del Río Santa Cruz. Las colecciones de Punta Entrada y Parque Nacional Monte León (Provincia de Santa Cruz, Argentina). En II Encuentro Latinoamericano de Zooarqueología. Santiago: Universidad de Chile.

Cabrera, A. L. (1971). Fitogeografía de la República Argentina. Boletín de la Sociedad Argentina de Botánica, XIV (1-2), 1-42.

Caggiano, M. A. (1977). La práctica de la pesca por arponeo en el Delta del Paraná. Relaciones de la Sociedad Argentina de Antropología XI, 101-106.

Capparelli, A.; A. Castro \& M. L. Ciampagna. (2009). Descripción macroscópica e identificación anatómica de un instrumento de madera (¿arpón?) Hallado en el sitio Cueva del Negro (costa norte de Santa Cruz Argentina) En M. Salemme, F. Santiago, M. Álvarez, E: Piana, M. Vázquez \& E. Mansur (Eds), Arqueología de la Patagonia, Una mirada desde el último confín. Ushuaia: Editorial Utopías.

Cárdenas R., Montiel, D., \& Grace Hall, C. (1991). Los Chono y los Veliche de Chiloé. Santiago: Ediciones Olimpo.

Carrara, I. S. (1952). Lobos marinos, pingüinos y guaneras de las costas del litoral marítimo e islas adyacentes de la República Argentina. Informe técnico, Facultad de Ciencias Veterinarias, Universidad Nacional de La Plata. Publicación especial de la Cátedra de Higiene e Industrias.

Castro, A. S., J. E. Moreno, M. Andolfo, R. Gimenez, C. Pe- ña, L. Mazzitelli, M. A. Zubimendi \& P. Ambrústolo. (2003). Análisis distribucionales en la costa de Santa Cruz (Patagonia Argentina): alcances y resultados. Anales del Instituto de la Patagonia, Serie Ciencias Humanas, 31, 69-94.

Castro, A., J. E. Moreno, M. A. Zubimendi, M. Andolfo, B. Videla, P. Ambrústolo, L. Mazzitelli \& S. Bogan. (2007). Cronología de la ocupación humana en la costa norte de Santa Cruz: actualización de datos radiocarbónicos. En F. Morello, M. Martinic, A. Prieto \& G. Bahamonde (Eds.), Arqueología de Fuego-Patagonia. Levantando piedras, desenterrando huesos... Y develando arcanos. Punta Arenas: Ediciones CEQUA.

Emperaire, J. (2002). Los nómades del mar. Santiago: LOM Ediciones.

Gaete, N., X. Navarro, F. Constantinescu, C. Mera, D. Selles, M. E. Solari, M. Loreto Vargas, D. Oliva \& L. Durán. (2004). Una mirada al modo de vida canoero del mar interior desde Piedra Azul. Chungara Revista de Antropología Chilena, 36 Supl. Especial, 333-346.

Gómez Otero, J., J. L. Lanata \& A. Prieto (1998). Arqueología de la Costa Atlántica Patagónica. Revista Americana de Arqueología 15, 107-185.

Legoupil, D. (1997). Bahía Colorada (île d'Englefield). Les premierschaussers de mammifèresmarins de Patagonieaustrale. Paris: Memoires de l'a.D.P.F., Recherches sur les Civilisations.

Legoupil, D. (2003). Cazadores- Recolectores de Ponsonby (Patagonia Austral) y su paleoambiente desde VI al III Milenio A. C. Magallania 31, número especial completo.

Lyman, R. L. (1994). Vertebrate Taphonomy. Cambridge: Cambridge University Press.

Mengoni Goñalons, G. (1999). Cazadores de guanacos de la estepa patagónica. Buenos Aires: Sociedad Argentina de Antropología, Colección Tesis Doctorales.

Miotti, L. (1990-1992). La experimentación simulativa de fracturas y marcas óseas y sus implicancias Arqueológicas. Arqueología Contemporánea 3, 39- 60.

Molina, M. J. (1967-1970). Arpones monodentados de la Patagonia Meridional. Acta Præehistórica VIII/X, 173-179.

Moreno, J. E. (2009). Arqueología e etnohistoria de la Costa Patagónica Central en el Holoceno Tardío. Chubut: Fondo Editorial Provincial, Secretaría de Cultura del Chubut.

Moreno, J. E., A. Castro \& F: Pepe. (2000). El rompecráneo: Un artefacto probablemente destinado a la caza de pinnípedos en la costa patagónica. En J. B. Belardi, F. Carballo \& S. Espinosa (Eds.), Desde el País de Los Gigantes. Perspectivas arqueológicas en Patagonia. Río Gallegos: Universidad Nacional de la Patagonia Austral. 
Orquera, L. A. (1987). Advances in the archaeology of the Pampa and Patagonia. Journal of World Prehistory 1(4),333-413.

Orquera, L. A. \& E. L. Piana (1995). Tunel VII: La Excavación. En J. Estévez \& A.Vila (Eds.), Encuentros en los conchales fueguinos. Barcelona: Universidad Autónoma de Barcelona, Serie Treballs D'Etnoarqueología, 1.

Orquera, L. A. \& E. Piana (1999a). La vida material y social de los Yámana. Buenos Aires: Instituto Fueguino de Investigaciones Científicas y EUDEBA.

Orquera, L. A. \& E. Piana. (1999b). Arqueología de la Región del Canal Beagle (Tierra del Fuego, República Argentina). Buenos Aires: Publicaciones de la Sociedad Argentina de Antropología.

Orquera L. A., A. E. Sala, E. L. Piana \& A. H. Tapia (1977). Lancha Packewaia. Buenos Aires: Editorial Huemul.

Ortiz Troncoso O. R. (1975). Los yacimientos de Punta Santa Ana y Bahía Buena (Patagonia Austral) Excavaciones y fechados radiocarbónicos. Anales del Instituto de la Patagonia 6, 93-122.

Outes, F. (1916). Sobre el hallazgo de un arpón de hueso en la región de Cabo Blanco (Gobernación de Santa Cruz). Physis II, 272-276.

San Román, M. (2005). Nuevos hallazgos de sitios de cazadores recolectores marinos tempranos en la Isla Englefield, Mar de Otway. Magallania 33(2), 173-176.

Scheinsohn, V. (1998). Explotación de materias primas óseas en la Isla Grande de Tierra del Fuego. Doctodo, Universidad de Buenos Aires.

Scheinsohn, V. (2005). Hunting at the end of the world: har- poon heads of Beagle Channel, Isla Grande de Tierra del Fuego (Patagonia, Argentina). Enviado a Links that Tie, editado por Krish Seetah. MS.

Schiavini, A., E. A. Crespo \& V. Szapkievich (1999). Estado de la población del lobo marino de un pelo (Otaria flavescens) en las provincias de Santa Cruz y Tierra del Fuego. Informes Técnicos del Plan de Manejo Integrado de la Zona Costera Patagónica, 40. Fundación Patagonia Natural.

Torres J. \& J. Ruz (2011). Pescadores de la tradición cultural Englefield. Datos preliminares en la zona del Estrecho de Magallanes, Chile. Magallania 39 (2), 165-176.

Vignati, M. A. (1930). Instrumental óseo aborigen procedente de Cabo Blanco (Gobernación de Santa Cruz) Notas del Museo etnográfico de la Facultad de Filosofía y Letras de la Universidad de Buenos Aires 3, 6-25.

Zubimendi, M. A., A. Castro \& J. E. Moreno (2004). Una aproximación hacia la definición de modelos de uso de la Costa Norte de Santa Cruz. Magallania 32,85-98.

Zubimendi, M. A., A. Castro \& J. E. Moreno (2005). Procesos de ocupación de la Costa Norte de Santa Cruz (Argentina). Relaciones de la Sociedad Argentina de Antropología XXX, 225-233.

Zubimendi, M. A., P. Ambrústolo, M. Beretta, L. Mazzitelli, M. L. Ciampagna, H. Hammond, L. Zilio, M. Plischuk \& A. Castro (2012). Sitio Cueva del Negro: Un caso de aprovechamiento intensivo de los recursos marinos en la Costa Norte de Santa Cruz (Patagonia Argentina). Revista de Estudios Marítimos y Sociales 4. En prensa. 
